\title{
A Pvdf Sensor for Monitoring Grain Loss in Combine Harvester
}

\author{
Jiaojiao $\mathrm{Xu}^{*}$ and Yaoming $\mathrm{Li}$ \\ Key Laboratory of Modern Agricultural Equipment and Technology, \\ Ministry of Education \& Jiangsu Province, Jiangsu University, P.R. China, 212013, \\ Tel.: +86-13952882634 \\ xujj525@163.com
}

\begin{abstract}
Grain loss sensor is an important monitoring module to realize the intelligent automatic control in combine harvester. Polyvinylidene fluoride piezoelectricity film has been one of the most widely used macromolecule materials at present. Based on its piezoelectric effect, in this paper, a grain loss monitoring system for combine harvester by using the PVDF sensor was designed. The system is composed of the PVDF sensor, intelligent display, SCM and so on. The working principle was analyzed and the measuring accuracy was dynamic calibrated in laboratory. The polyvinylidene fluoride piezoelectricity film was selected as the sensing material and the signal processing circuit was mainly composed by charge amplifier, band-pass filter. In order to verify the accuracy of the sensor, the dynamic calibration experiments were carried out. By adjustment of the feeder's vibration parameters, the different feeding velocity can be gained. In the dynamic calibration experiments, the sensor was fixed at the height of $19.5 \mathrm{~mm}$ and the fixing angle of $33^{\circ} \sim 35^{\circ}$, the feeding velocity of $60 \sim 80$ grain per second, 1000samples of full grain were randomly selected in the experiments, and the results indicated the detecting error was less than $5.7 \%$. Using the different mixture ratio of the full grain, the imperfect grain, short stalk to do multi-group experiments, the result shows that the sensor can distinguish the grain from the mixture stalks. In the rig-tests, the monitoring sensor was fixed on the caudal region of the cylinder concave grid of the combine harvester in order to monitor the threshing compounds. The precision error of the monitoring sensor in combine is less than $8 \%$ through field experiments. So this advanced sensor can realize the aim of monitoring the entrainment loss in combine harvester.
\end{abstract}

Keywords: PVDF, Grain Loss Sensor, Accuracy Calibration, Combine Harvester.

\section{Introduction}

Polyvinylidene fluoride (PVDF) is a new type of piezoelectric polymer material which has many advantages such as high piezoelectric voltage constant, light weight, low density, better toughness, wide frequency response, low acoustic impedance,

\footnotetext{
* Corresponding author.
} 
better sensitivity and stability, easy processing and installation(e.g., Xu Hongxing et al.,1999; Ju dianshu et al., 2004). The piezoelectric constant d of PVDF is higher than quartz as the value of more than 10 times and the value $\mathrm{g}$ is 20 times higher than PZT, while the low acoustic impedance is about $3.5 \times 10^{-6}$ which is only $1 / 10$ of the PZT piezoelectric ceramic. The frequency response is wide at room temperature in the range of $10-5 \sim 109 \mathrm{~Hz}$ and the response is flat. It means that from the quasi-static, low frequency, high frequency, up to ultra-high frequency ultrasound can convert mechanical and electrical effects. So it can be processed into thin films and be used as sensors, because the vibration response of the system has little effect.

Based on its piezoelectric effect, in this paper a set of monitoring system which can monitor the gain loss in combine harvester by using the PVDF sensor was designed. The monitoring system is composed by the PVDF sensor, intelligent display and so on. The working principle and measuring accuracy was analyzed and dynamic calibrated in laboratory.

\section{Dynamic Calibration Experiment}

\subsection{The Measuring Principle of PVDF Sensor}

Polyvinylidene fluoride is a kind of organic macromolecule polymers. Through heating, cold stretching and processing in the Curie point of temperature in order to demonstrate the polarization piezoelectric and thermoelectric properties. The pressure principle of PVDF piezoelectric films as shown in Figure 1 (e.g., Cheng Qihua and Li yongxin, 2007), the direction of uniaxial tension is the $\mathrm{x}$-axis, the $\mathrm{z}$-axis is perpendicular to the polarization surface and parallel to the polarization direction, the $y$-axis is perpendicular to the $\mathrm{x}$-axis and z-axis. When the PVDF membrane is acted by external force, the corresponding charge will appear on both sides of the film and the charge will led by the metal conductive layer. By measuring the amount of charge, it can achieve the measurement of pressure. In practice application (refer as Eq. (1)) only on the axial stress the relationship is between charge and plane (e.g., Tomasz Janiczek, 2001).

$$
q(t)=q\left(t_{0}\right)+\iint_{\Omega}\left[\int_{0}^{t}\left(\sum_{j=1}^{3} d_{3 j} \frac{\partial \sigma_{j j}(x, y, t)}{\partial_{t}}\right) d_{t}\right] d x d y-\int_{0}^{t} \frac{q(t)}{R C} d t
$$

Where: $q\left(t_{0}\right)=$ initial voltage, V. $d_{3 j}=$ piezoelectric constant, pc/N

$$
\sigma_{j j}(x, y, t)=\text { stress }
$$

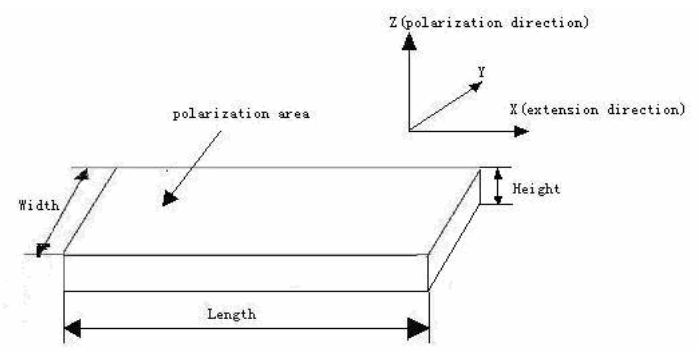

Fig. 1. Principle diagram of pressure measurement on PVDF 


\subsection{Design of Dynamic Calibration Experiment}

In the dynamic calibration experiments, the piezoelectric sensor is used by 5 sheets of PVDF film that each module's thickness is $50 \mathrm{~mm}$ and the size is $20 \mathrm{~mm} * 100 \mathrm{~mm}$. Fig. 2 is the circuit schematic diagram of single channel.

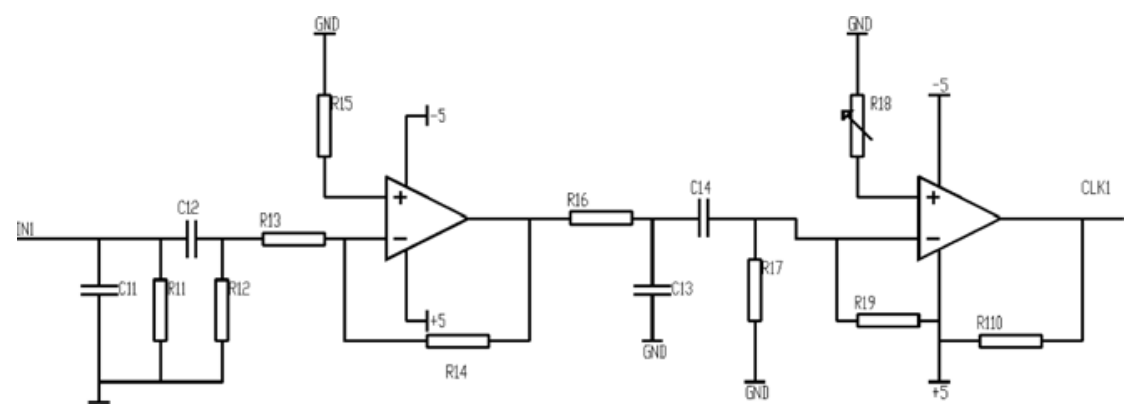

Fig. 2. Circuit schematic diagram of single channel

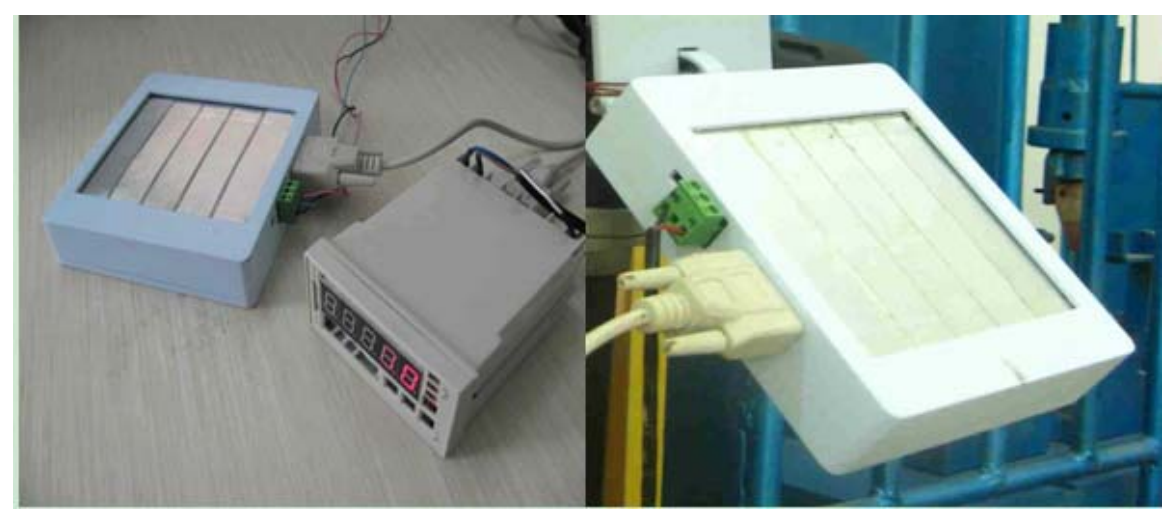

Fig. 3. Design of the monitoring sensor

The circuit schematic diagram includes filter circuit, frequency discriminating circuit, amplification system and shaping circuit. Fig. 3 is the design of the monitoring sensor. Because the whereabouts of threshing mixture has different height, the different contact forces for the sensor can cause different frequency response. So the sensor is installed on the bracket which the fixing height can be adjustable in order to regulate the whereabouts of a suitable calibration height though the contact forces on the sensor. The installing angle of sensor at the same time can also be adjusted and the different seeds, stem and others film has different impact forces on the PVDF piezoelectric (e.g., Li Junfeng, 2006). Therefore different angles must be adjusted to determine the optimum installation angle. The impact situation of the threshing compound in combine harvester was simulated using a magnetic vibration-actuated feeder. When the threshing grain and other compound drop on the sensor's film, the impact signals can be detected through the data wire and the amount of grain can be 
shown in the computer. Using the different mixture ratio of the full grain, the imperfect grain, short stalk to do multi-group experiments, the result shows that the sensor can distinguish the grain from the mixture stalks. Fig.4 is the Circuit structure of monitoring sensor.

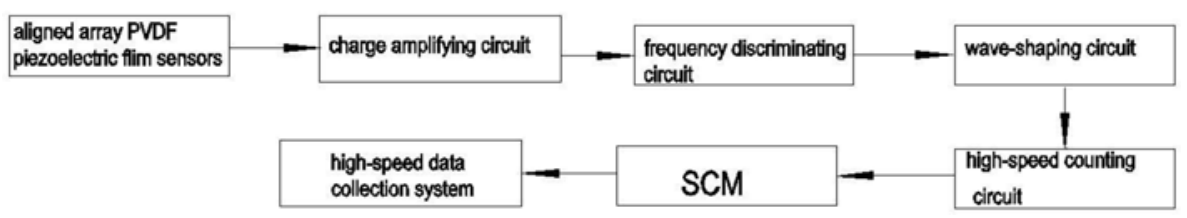

Fig. 4. Circuit structure of sensor

\subsection{Results of Dynamic Calibration Experiment}

When the different threshing mixture including grains, stalks and others drop on the sensor's surface, as the grain and the stalk has different impact force on the sensor's surface, so the different frequency and amplitude of the signal will be reflected in the oscillograph. Figure 5 shows the signal maps that the impact forces of grain and straw on the PVDF sensor's surface. As a result that miscellaneous residual are so light that when they fall on the sensor's surface, they don't has any trigger signals and thus they don't have any significant impact signal.
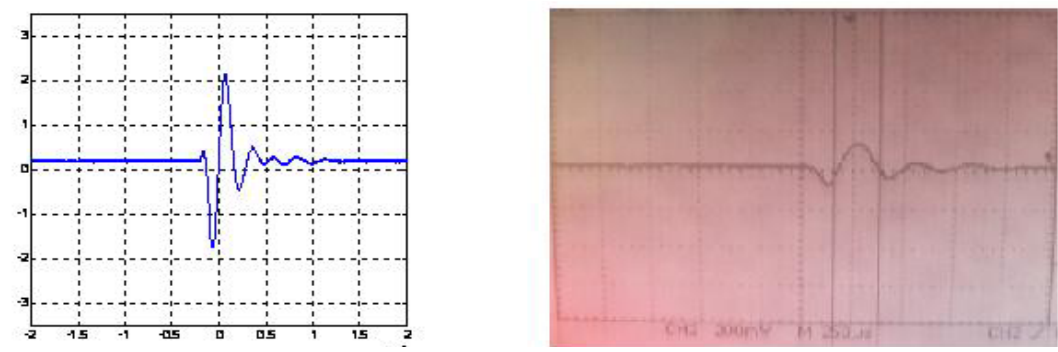

Fig. 5. The impact signal of grains and straws

The abscissa axis is time and the vertical axis is voltage. So in the proceedings, the cutoff frequency of band-pass filter and the amplitude threshold voltage of the frequency discriminator must be reasonable set. And the chaotic signals can be extracted from the impact of grain, then the signals will be counted by program and they can be accurately recorded the amount of grain losses.

\section{Analysis of Experiments Results}

\subsection{Analysis of Dynamic Calibration Experiment}

In dynamic calibration experiments, different moisture content of rice grains and different length of straws are used as the calibration materials. The moisture content are 
respectively $14.72 \%, 18.32 \%, 23.19 \%$. The same moisture content of $39.33 \%$ has different length of straws. The lengths are $10 \mathrm{~mm}, 20 \mathrm{~mm}, 30 \mathrm{~mm}$. The thousand-grain weight of the full grains is $28 \mathrm{~g} \sim 32 \mathrm{~g}$. The sensor was fixed on a bracket which can adjust its height and the sensor's fixing angle was also can be adjusted.

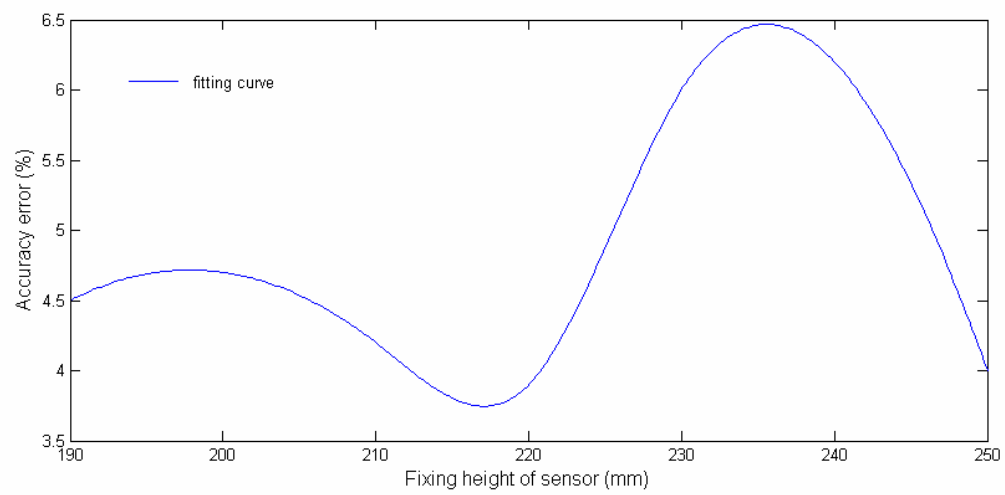

Fig. 6. Calibration curve of variable height

The impact situation of the threshing compound in combine harvester was simulated using a magnetic vibration-actuated feeder. When the threshing grain and other compound drop on the sensor's film, the impact signals can be detected through the data wire and the amount of grain can be shown in the display. As shown in Figure 6, the calibration curve is obtained. When the sensor's fixing height between $190 \mathrm{~mm}$ and $225 \mathrm{~mm}$, the sensor can meet the testing requirements (e.g., Wan Jianguo et al.,1998).

Similarly, as shown in Figure 7, from the calibration curve, when the reasonable fixing angle between $32^{\circ}$ and $43^{\circ}$ the sensor can meet the testing requirements and the accuracy error is steady.

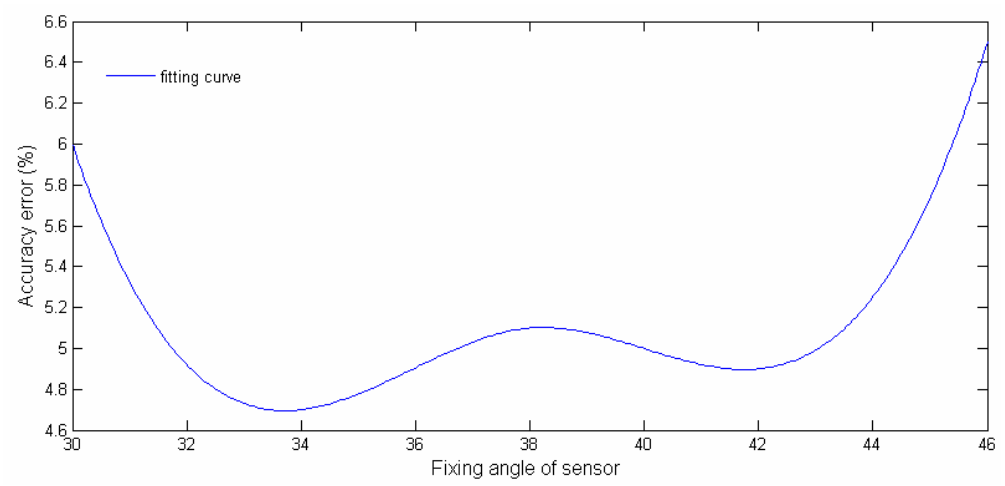

Fig. 7. Calibration curve of variable angle 


\subsection{The Fixing Position of Sensor in Rig-Test}

The monitoring sensor can be fixed under the combine threshing cylinder to collect the grain signals from threshing mixture. Figure 8 shows the actual view of sensor's installing place. The sensor can be installed under the separation device at three positions .Figure 9 shows the left view of sensor, the sensor installs on the bracket and the location is at the end of concave. The threshing probability curve and mathematical model are established through the threshing and separation process along the axial points of the combine cylinder. In order to find out the mathematical relationship between the amount grains monitored from the sensor and the loss grains from the rowing straw port, the high-speed data acquisition system collect the amount grains and using the mathematical model in order to get the entrainment losses in the harvest process.

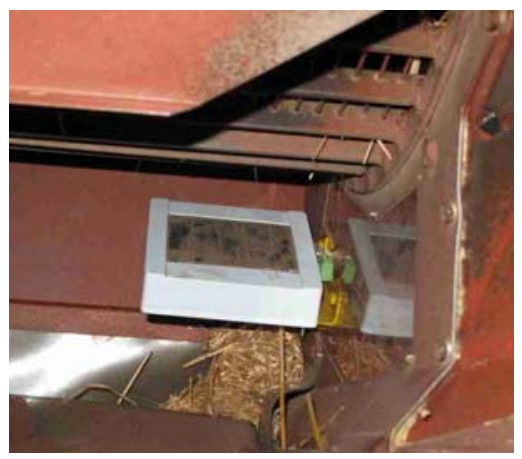

Fig. 8. Sensor's installation position

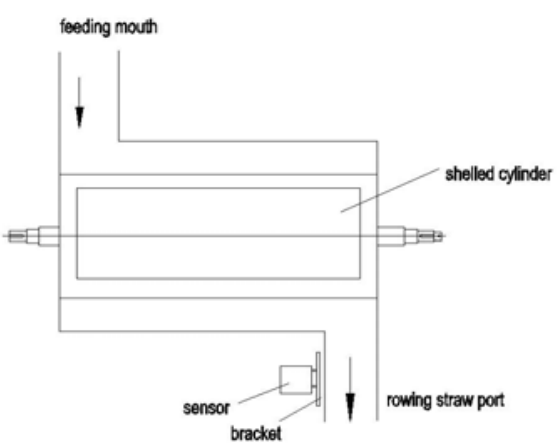

Fig. 9. Left view of the installation position

\section{Conclusion}

Through bench tests and calibration experiments, the monitoring sensor can detect the amount of grain losses. The monitoring system bases on sensor techniques and computer technology and the mathematical model of science as a guide in order to achieve the monitoring of entrainment losses. Fundamentally this method and device change the traditional manual method of testing entrainment losses and it can save manpower, material and eliminate the non-real-time test of entrainment loss. At the same time this method and device is propitious for the driver to monitor the process of entrainment loss during the combines' work status in real-time and achieve the automatic control of operating parameters in order to improve the efficiency of the combine harvester.

\section{Acknowledgements}

This work was supported by the National Key Science \& Technology Pillar Program during the Eleventh Five-Year Plan Period (2006BAD11A03). 


\section{References}

Qihua, C., Yongxin, L.: Application of PVDF sensor to the collision stress detection on the projectile base of artillery. Journal of Test and Measurement Technology 21(1), 90-94 (2007)

Dianshu, J., Zhi, Z., Jinping, O.: Study on strain-sensing of PVDF films. Journal of Functional Materials 4(35), 450-456 (2004)

Junfeng, L.: Improvement design of the structure of combine harvester grain loss sensor and laboratory calibration. Agricultural equipment \& vehicle engineering 184(11), 10-13 (2006)

Janiczek, T.: Analysis of PVDF transducer signals stimulated by mechanical tension. Journal of Electrostatics 51(52), 167-172 (2001)

Jianguo, W., Jijun, Z., Lihua, S.: Preliminary search on application of PVDF piezoelectric film for monitoring structures. Chinese Journal of Sensor and Actuators 12(1), 18-24 (1998)

Hongxing, X., Zuting, L.: Developments of PVDF Piezoelectric Films. Journal of Jiangsu University of Science and Technology 20(5), 88-91 (1999) 